\title{
Buckling and Post-Buckling Behavior of Unitized, Stiffened Tri-Axially Braided Composite Textile Plates
}

\author{
Cyrus J. R. Kosztowny ${ }^{1}$ and Anthony M. Waas ${ }^{2}$ \\ Composite Structures Laboratory, Department of Aerospace Engineering \\ University of Michigan, Ann Arbor, MI, 48109
}

\begin{abstract}
This investigation examines the buckling and post-buckling response of unitized, doubly stiffened textile fiber reinforced composite plates using the finite element method, and in conjunction with the commercial code Abaqus. Investigations into the global buckling mode shapes, and corresponding loads, and significant behavior during loading in the postbuckled regime are also of significant importance. A two dimensional, tri-axially braided (2DTBC) textile is used to manufacture the panels, and a new approach to creating the stiffener geometry is presented. How the new stiffener formation alters the buckling, post-buckling, and failure response is examined. Axial compressive loading is considered, and computational results are provided and compared against a set of limited experimental data.
\end{abstract}

\section{Introduction}

Thin-walled structures are widely used in aerospace applications due to sufficient in-plane performance while reducing overall structural weight. Common techniques to further improve structural performance include increasing the second moment of area (ex. sandwich structures), adding significant internal supports (truss structures), or attaching stiffeners (combination of the two) ${ }^{1}$. When loading under compression beyond the buckling limit, however, stiffeners are prone to debonding in metallic structures or they can delaminate in pre-preg tape based composite structures. Once the stiffener separates from the face sheet, there is a significant loss in load carrying capacity of the structure ${ }^{2}$. This negative feedback cycle often results in a sudden and catastrophic collapse of the structure.

Work has been done to reduce the tendency for stiffener separation through composite pre-form modifications like stitching the stiffener to the face sheet ${ }^{3}$. The new approach to creating the stiffener geometry used in this investigation utilizes the textile nature of the 2DTBC to make the stiffeners an extension of the face sheet. A textile layer is folded over aluminum molds onto itself as a continuous piece to create the stiffener geometry, yet the layer is incorporated into the formation of the face sheet as well. As a result, the stiffener is integrally manufactured as a part of the face sheet and the number of interfaces between the stiffener and face sheet is reduced. For the stiffener to separate from the face sheet, either the entire textile layer needs to delaminate or the carbon fiber tows need to fracture and split into two pieces. Either of these two methods are far more energy intensive than the energy required to delaminate a traditionally bonded stiffener ${ }^{4}$. The increased energy needed to fully separate the stiffener could result in two predicted behaviors: either the unitized, stiffened panel will gradually fail rather than catastrophically fail, or another failure mode will initiate before complete stiffener delamination occurs 5 .

\section{Stiffened Panel Description}

For this investigation, dry textile 2DTBC was infused with Epon 862/EpiKure 3234 epoxy/hardener system using a Vacuum-Assisted Resin Transfer Molding (VARTM) method. Figure 1 shows a representation of the triaxial braid textile.

\footnotetext{
${ }^{1}$ NASA Space Technology Research Fellow, Department of Aerospace Engineering, 1320 Beal Avenue, Ann Arbor, MI, 48109, AIAA Student Member.

${ }^{2}$ Felix Pawlowski Collegiate Professor of Aerospace Engineering, Department of Aerospace Engineering, 1320 Beal Avenue, Ann Arbor, MI, 48109, AIAA Fellow.
} 

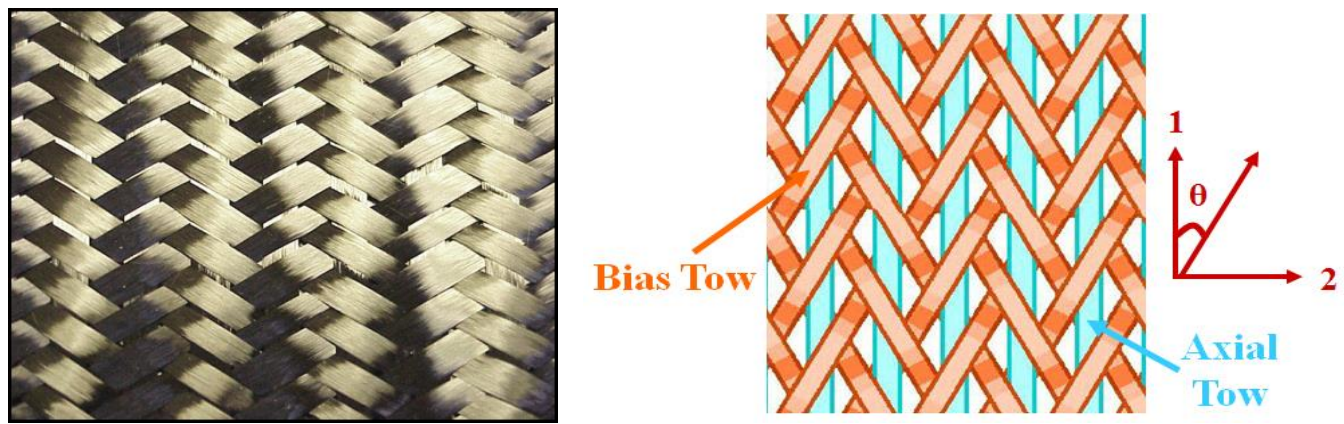

Figure 1. Photo of $\pm 60^{\circ}$ TBC textile (left) and a diagram of the braided axial and bias tow architecture (right).

The VARTM method was preferred over traditional vacuum bagging methods such as wet layup or using high pressure systems due to the stiffener geometry and product consistency within the lab setup. The vacuum pressure helps ensure even fiber wetting regardless of mold orientation during the resin infusion process, and it helps consolidate the mold to achieve a panel with little deviation between samples when a systematic manufacturing procedure is followed. Inverted L-shaped doubly stiffened panels were manufactured from $\pm 30^{\circ}$ and $\pm 60^{\circ} \mathrm{TBC}$ textiles. Four lamina were used in the unitized, stiffened panels, and all lamina in a panel were the same $\pm \theta^{\circ} \mathrm{TBC}$ textile. Two lamina create half of the face sheet, while the other two lamina are folded over to create the remaining thickness of the face sheet as well as the stiffener geometry. Though only two lamina were used to make the stiffeners, the textile folds over onto itself to create a four lamina thick stiffener. Figure 2 shows the final panel after trimming and removal from the mold.

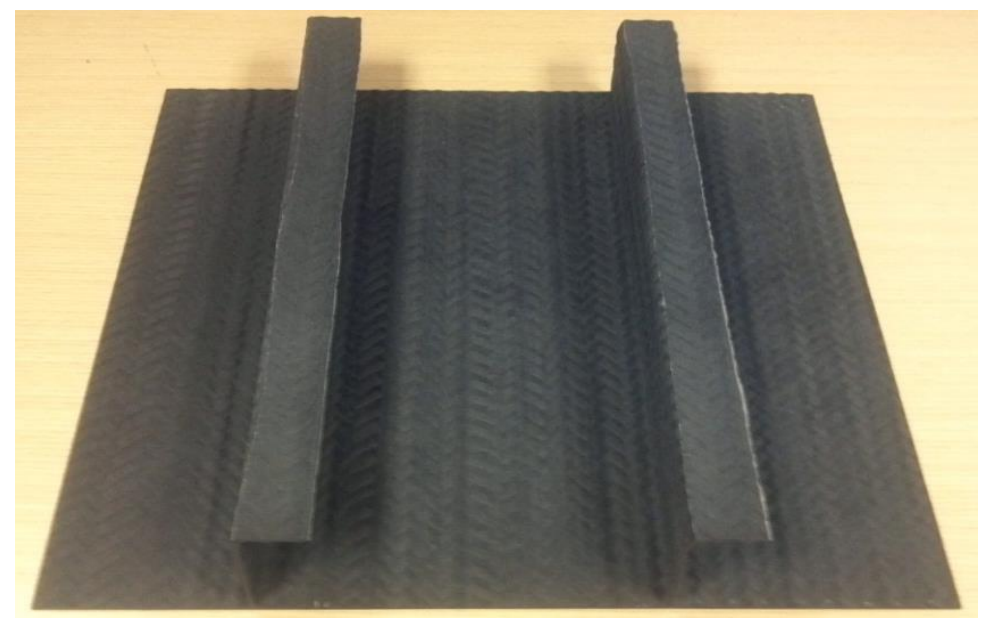

Figure 2. Trimmed and squared panel prior to potting in steel putty and aluminum blocks for experimental testing.

The panels are approximately 267 millimeters long by 280 millimeters wide before potting both ends in $19 \mathrm{~mm}$ deep channels. Each stiffener has a web approximately $40 \mathrm{~mm}$ tall and a $20 \mathrm{~mm}$ wide flange to create the inverted Lshaped geometry. The thickness is approximately $4 \mathrm{~mm}$ for the face sheet and stiffeners. Steel putty encases the ends of the panels to create effectively clamped end conditions ${ }^{6}$. Figure 2 shows a diagram of the nominal unitized, doubly stiffened TBC panels. Note that the diagram in Figure 2 is not to scale. 


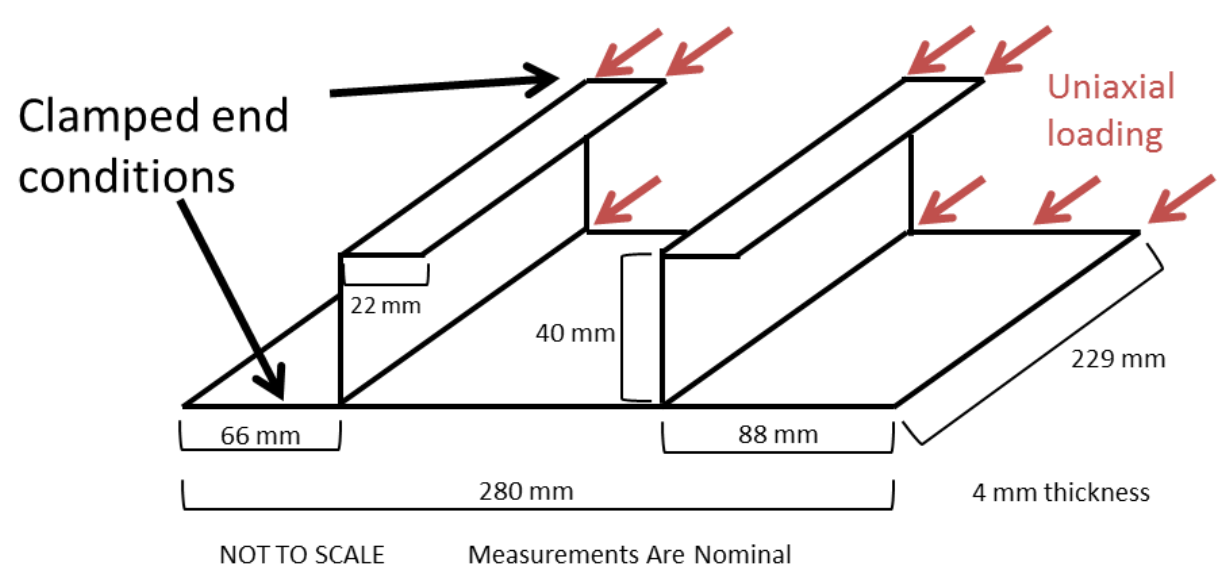

Figure 3. Diagram of nominal geometry for a unitized, stiffened TBC panel as modeled in Abaqus.

\section{Modeling}

A finite element model of the panel was developed and analyzed using the commercial software package Abaqus to accurately capture the pre-buckling stiffness, approximate buckling limit, and the initial post-buckling stiffness behavior. The material is assumed to be linear elastic and orthotropic, with the homogenized properties calculated using the procedure described in Ref. 5. S4R shell elements are used in the model. Note that while the constituents (TBC fiber and matrix) are the same as in Ref. 5, the VARTM process changes details like the fiber volume fraction and tow packing arrangement. The VARTM process consolidates the fibers more than the specimens used in Ref. 5, so the fiber volume fraction is expected to be higher. While a full characterization of the VARTM material as used is planned, the material properties from Ref. 5 are considered adequate for the initial modeling efforts. The geometry and boundary conditions given in Figure 3 were applied in the model.

\section{A. Buckling Analysis}

A linear buckling analysis (eigenvalue problem) was performed to obtain the first few buckling modes. The panel ends are clamped and the lateral sides of the panel are free. Figure 4 shows the first 8 buckling modes for the $\pm 30^{\circ}$ and $\pm 60^{\circ} \mathrm{TBC}$ unitized, stiffened textile panels along with corresponding buckling loads normalized against the first mode load. A displacement towards the reader is colored red, while a displacement away from the reader is colored blue.
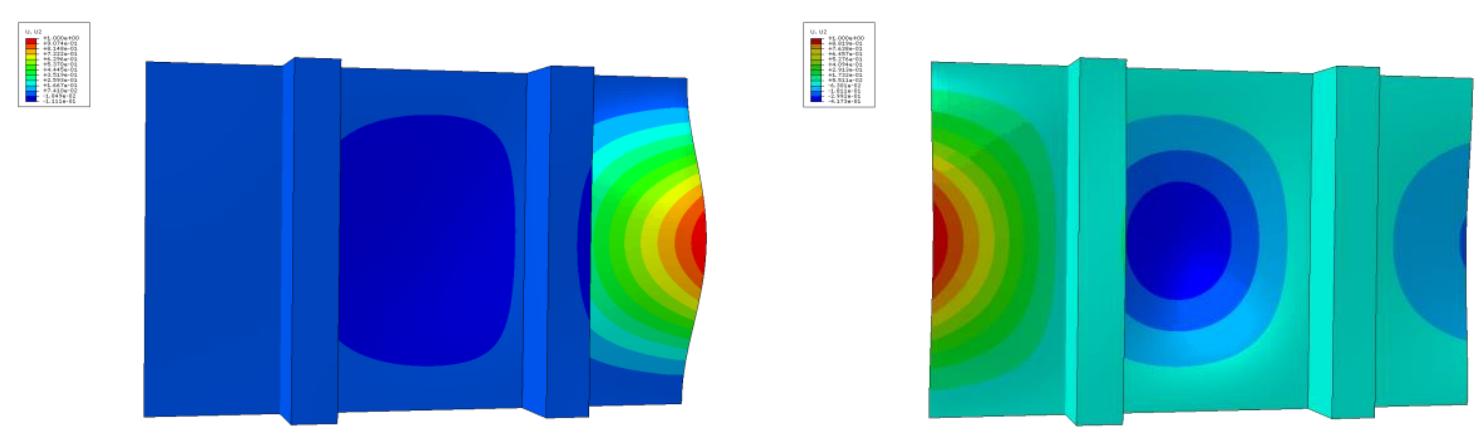

TBC 30 Mode 1 Normalized Buckling Load: 1.000

Mode 2: 1.290 


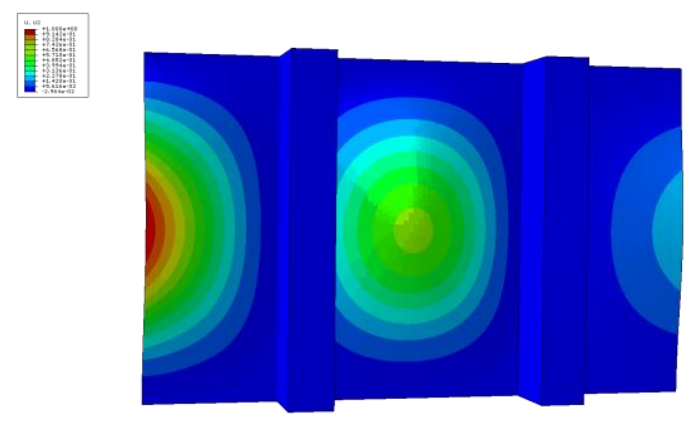

Mode 3: 1.523

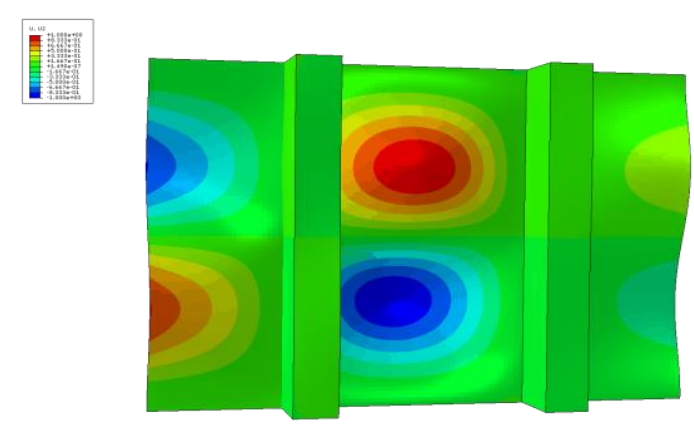

Mode 5: 1.805

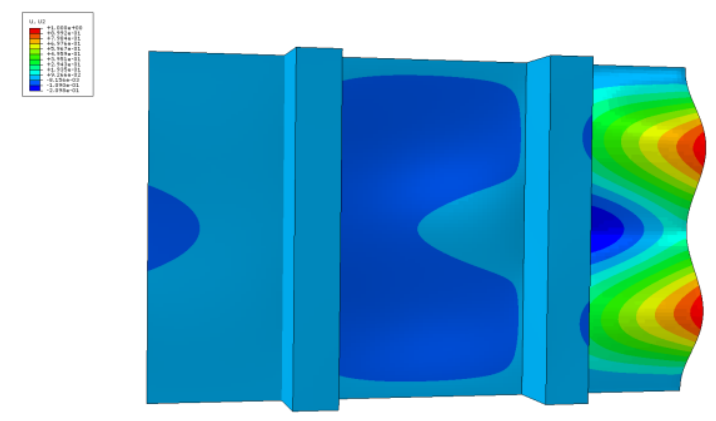

Mode 7: 2.786

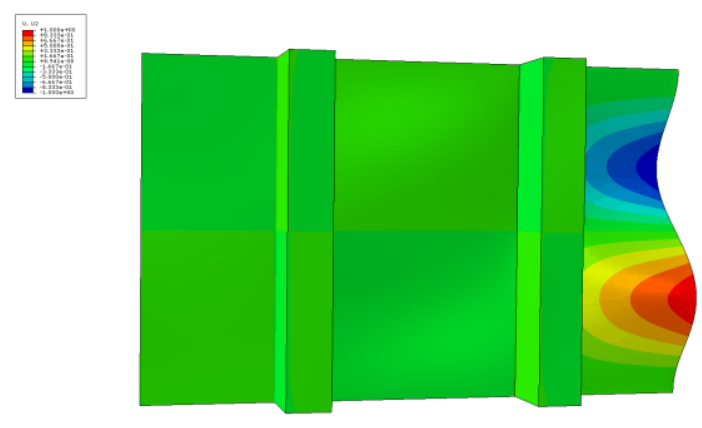

Mode 4: 1.630
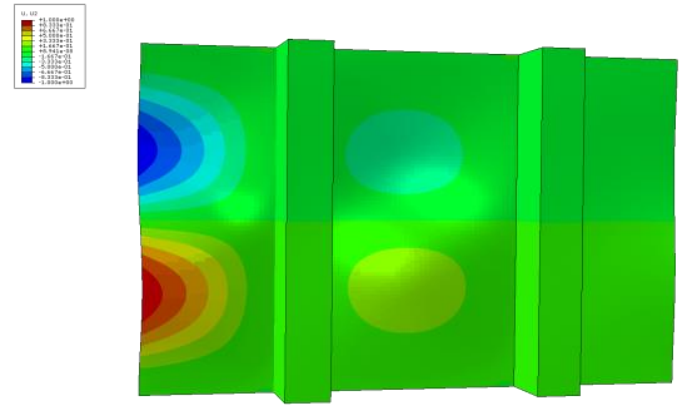

Mode 6: 1.928
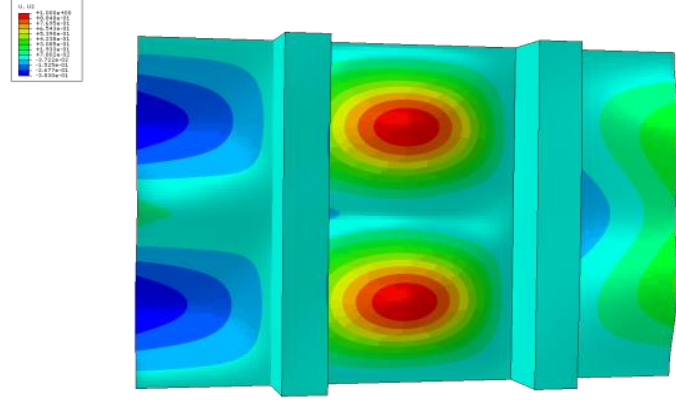

Mode 8: 2.908

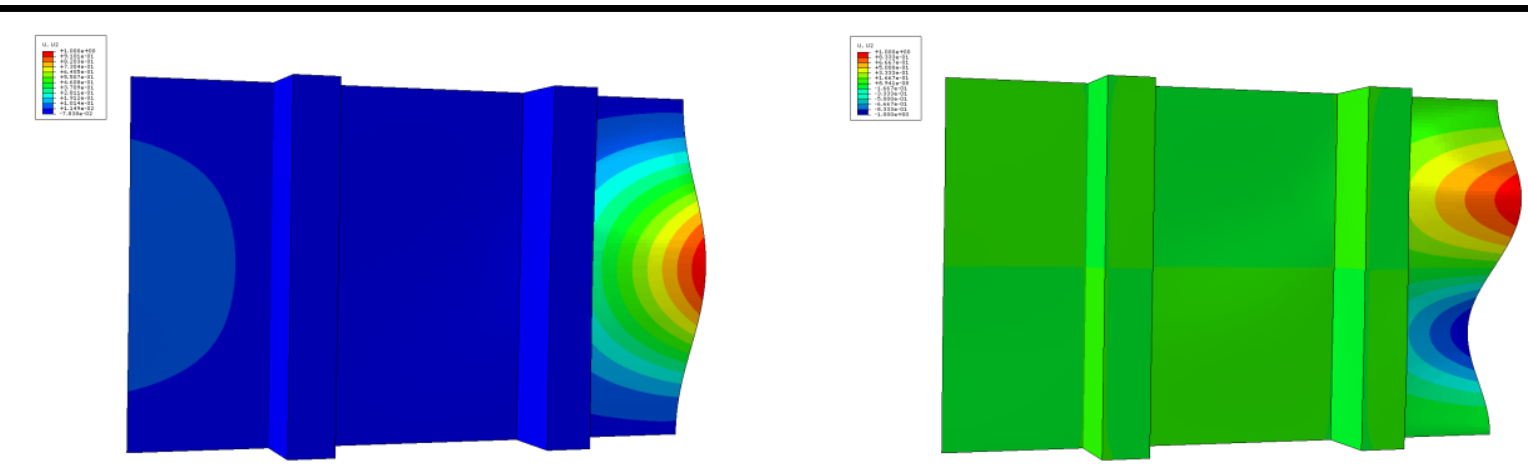

TBC 60 Mode 1 Normalized Buckling Load: 1.000

Mode 2: 1.269

4

American Institute of Aeronautics and Astronautics 


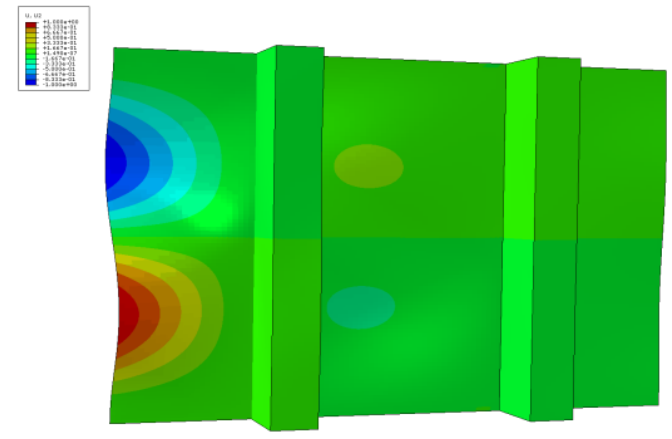

Mode 3: 1.627

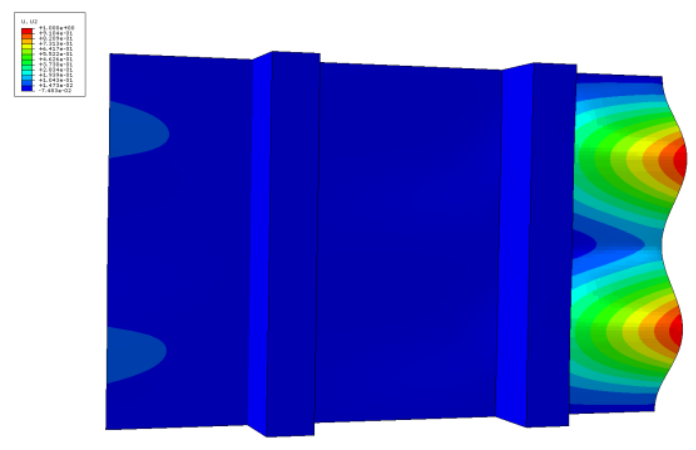

Mode 5: 2.048

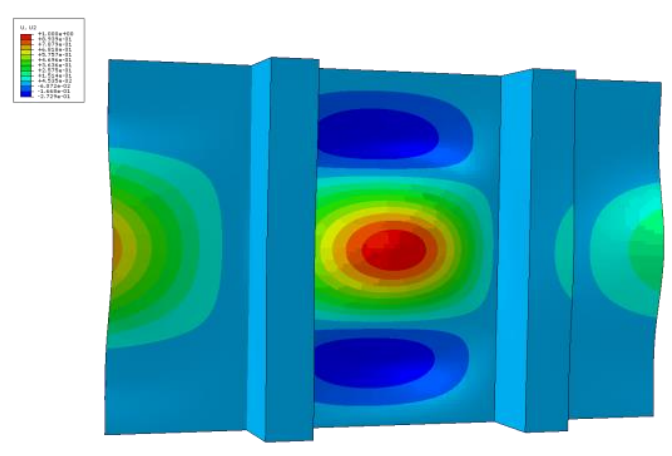

Mode 7: 2.265

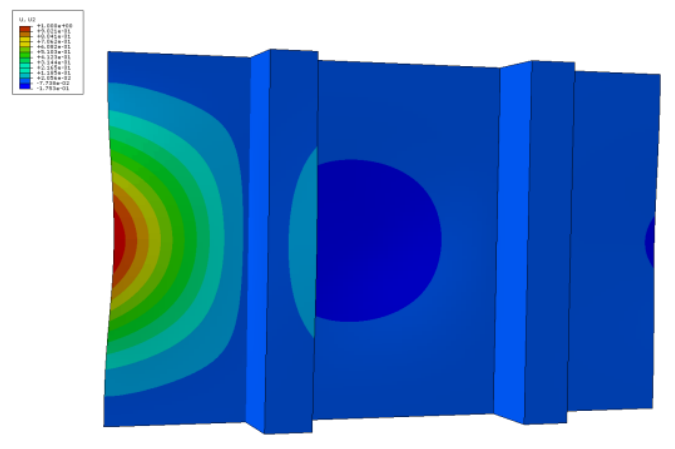

Mode 4: 1.635

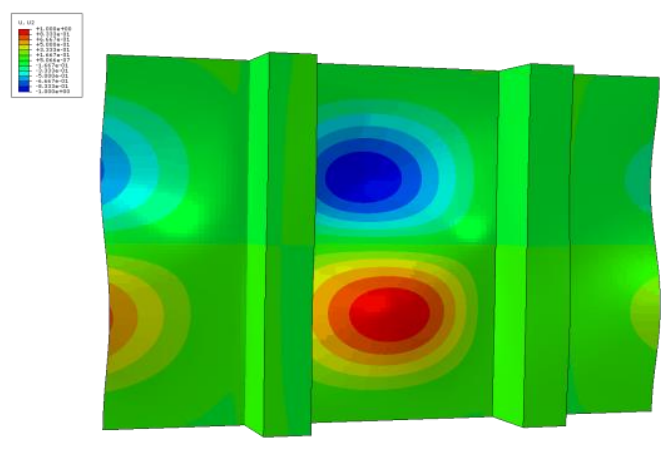

Mode 6: 2.052

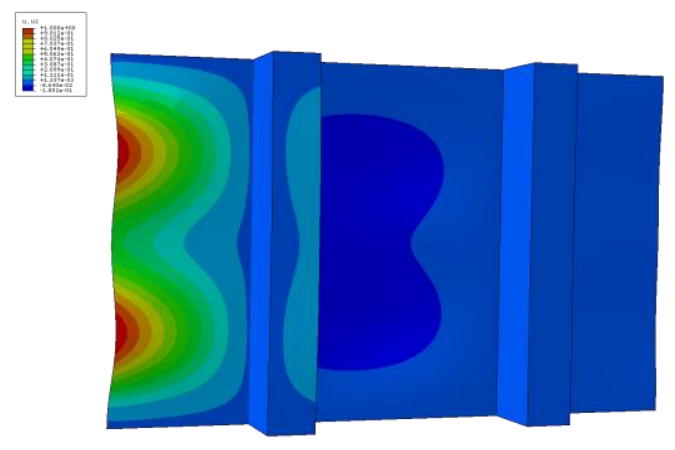

Mode 8: 2.412

Figure 4. Buckling modes and normalized loads for the TBC 30 and TBC 60 stiffened panels.

While the first mode shape for both the ТВC 30 and ТВC 60 panels are similar, further similarities cease at higher modes. For both types of panels, a single half wave occurs in the wider unsupported edge section. The load at which the first mode is predicted is 49,855 Newtons for TBC 30 and 34,893 N for TBC 60 . 


\section{B. Nonlinear Response Analysis}

A general, static step with nonlinear geometry was created for the TBC 30 model and seeded with an initial global imperfection of the first buckling mode with amplitude of $10 \%$ of the panel thickness. Figure 6 shows the initial post-buckling load-displacement behavior. This pre- and post-buckling step was run until a specified value of compressive strain was obtained. The average of the bending strains at opposing surfaces were used to determine the overall compressive strains. Figure 6 shows the load-displacement curves for both the prediction and an experimental test of a TBC 30 panel.

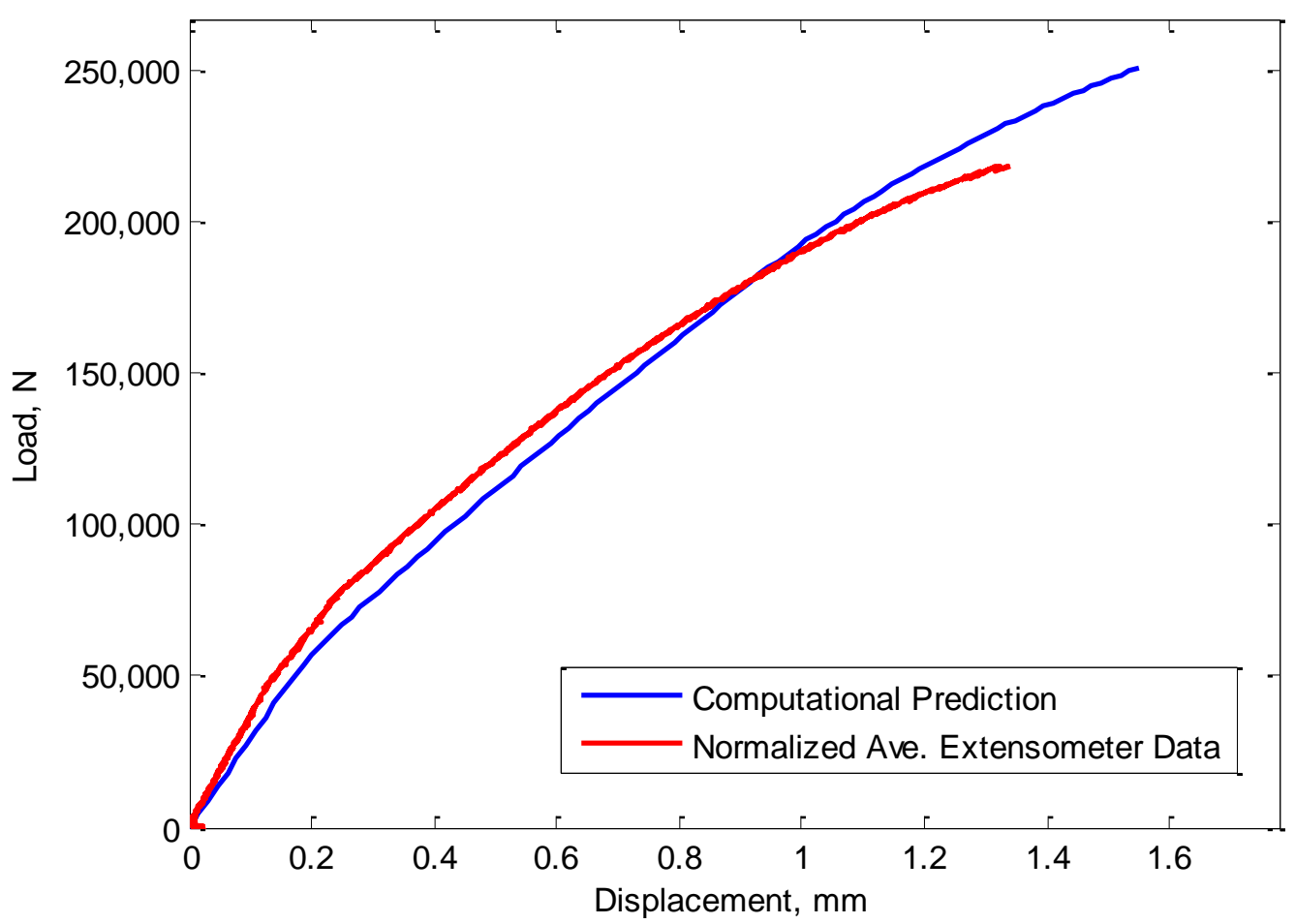

Figure 6. Load-displacement curves for a unitized, stiffened $\pm 30^{\circ}$ TBC panel without damage or failure modeling.

A digital imaging correlation (DIC) system was used to gather the experimental displacement data, normalized over the full length of the specimen, and averaged across 3 virtual extensometer locations. While the initial predicted stiffness is slightly less than experimentally observed, the aforementioned VARTM manufacturing consolidates fibers more and could lead to the slightly increased stiffness. The experimental buckling load is $46,306 \mathrm{~N}$ compared to the predicted $49,855 \mathrm{~N}$, and the strain-limit load is $218,400 \mathrm{~N}$ compared to the predicted $251,100 \mathrm{~N}$. Use of a nonlinear material description or damaging material is believed to increase the accuracy of the postbuckling stiffnesses and strain-limit prediction.

Strain gages on the physical specimen were attached according to the layout in Figure 8. Ten pairs of back-toback strain gages were used to capture the response during compression in addition to the DIC system. Gages 11/12, 13/14, and 15/16 located along the skin midline are presented in the top graph in Figure 9, while the computational equivalent gages are shown in the bottom graph in Figure 9. 

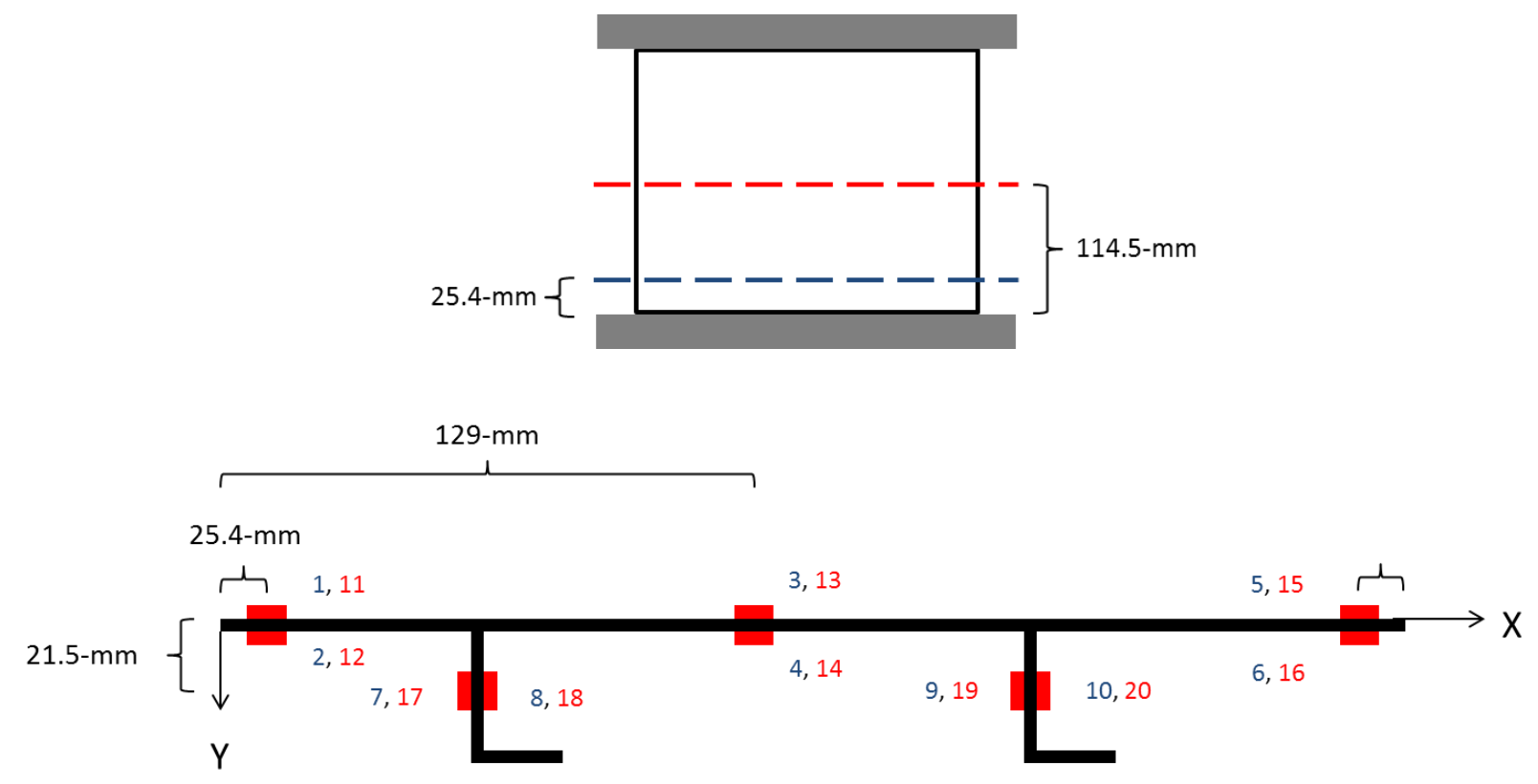

Figure 8. Two rows of gages (top), red being along the midline of the specimen. Location of the back-to-back pairs (bottom) with the corresponding numbering scheme used in Figure 9.

The qualitative behavior between graphs in Figure 9 shows promising agreement. However, there are small details that require further model development and is part of ongoing work which will consider experimental data from more panels. One example is that all experimental strains have a higher load at a given strain value. A solution to this could again be the VARTM process resulting in an expected higher fiber volume fraction compared to the material used in Ref. 5. Another is the delay in which experimental pairs 11/12 and 13/14 diverge well after the pair $15 / 16$ while the computational pairs diverge at approximately equivalent times.

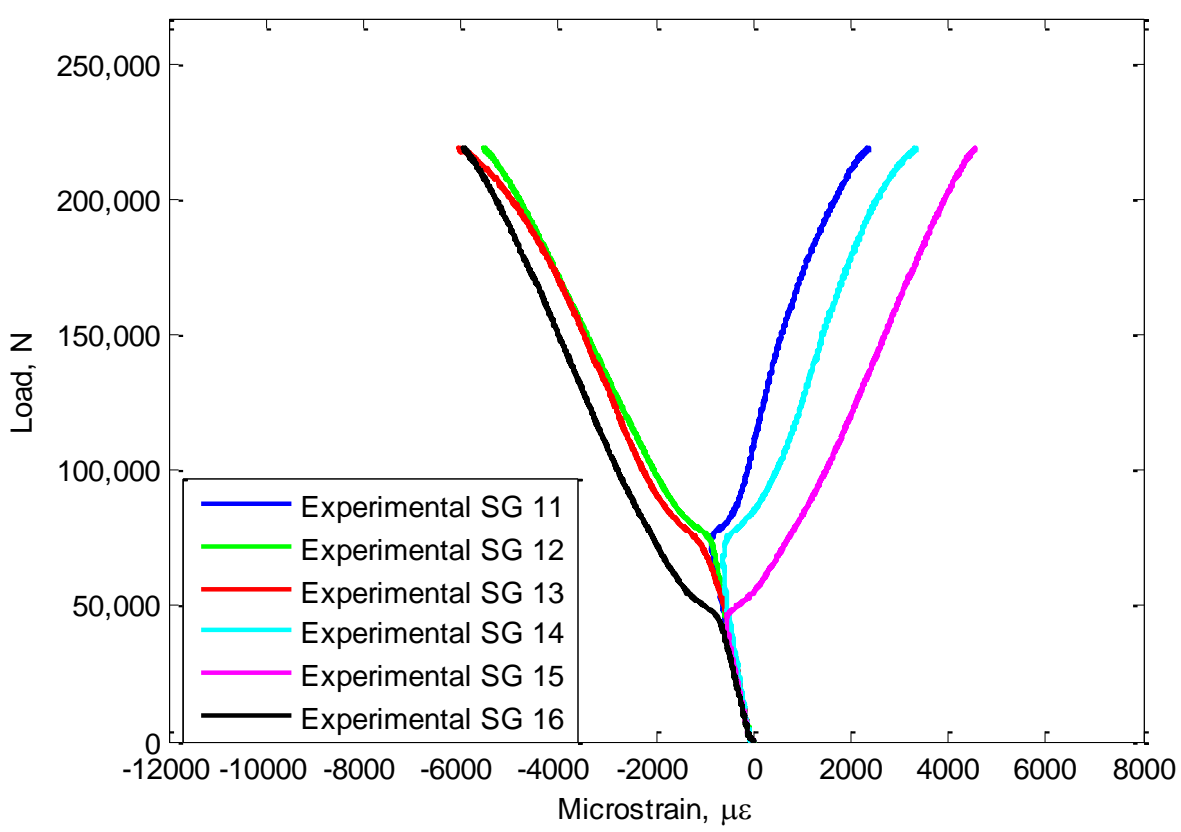

American Institute of Aeronautics and Astronautics 


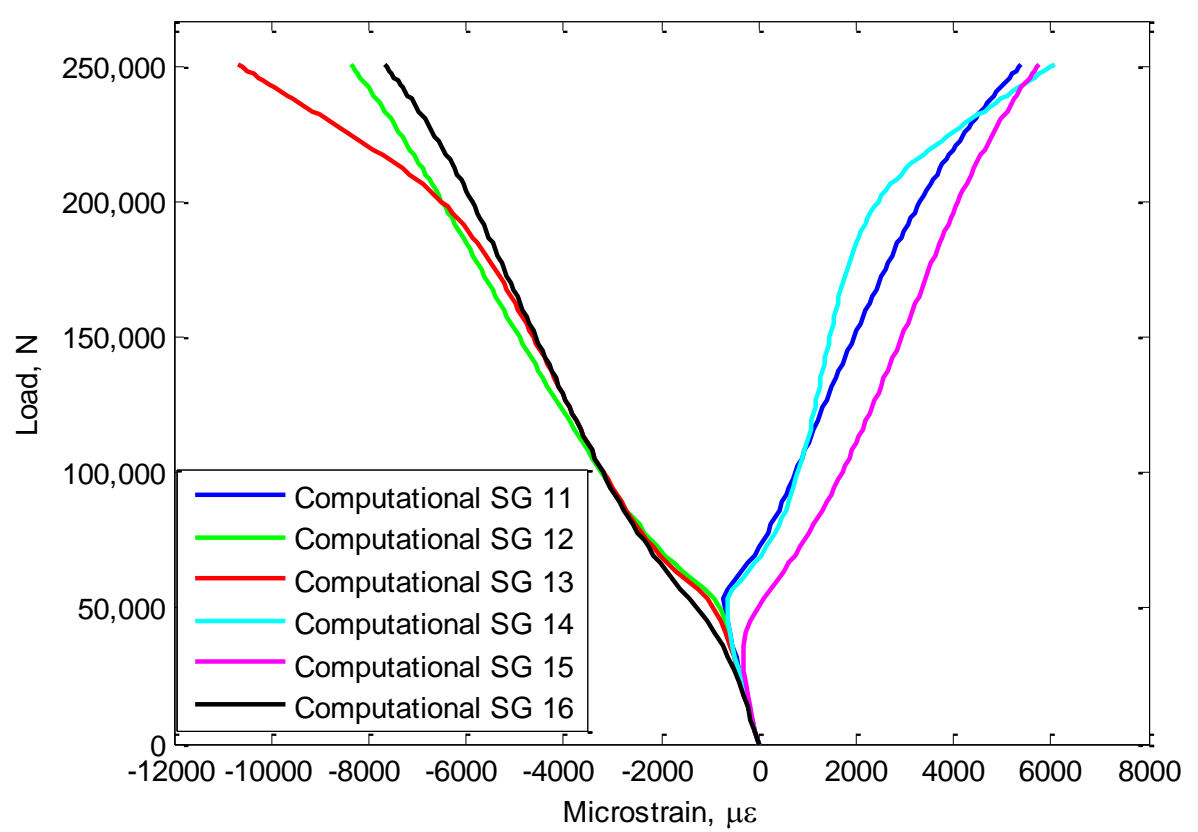

Figure 9. Experimental strains and corresponding computational strains for the TBC 30 specimen.

For modeling the TBC 60 panel, a static Riks arc-length $\operatorname{method}^{7}$ was used with nonlinear geometry. A Riks step was needed to achieve convergence on imperfection seedings with imperfection magnitudes of less than $8 \%$ of the thickness. During the corresponding experimental test, a mode switch was observed. The mode switch from the first to second mode was not captured using a single imperfection mode seed, so the second mode shape was used at $1 \%$ skin thickness in addition to the first mode shape imperfection. Figure 10 shows the initial post-buckling loaddisplacement behavior without any damage or failure models. This pre- and post-buckling step was ran until a specified value of compressive strain was obtained.

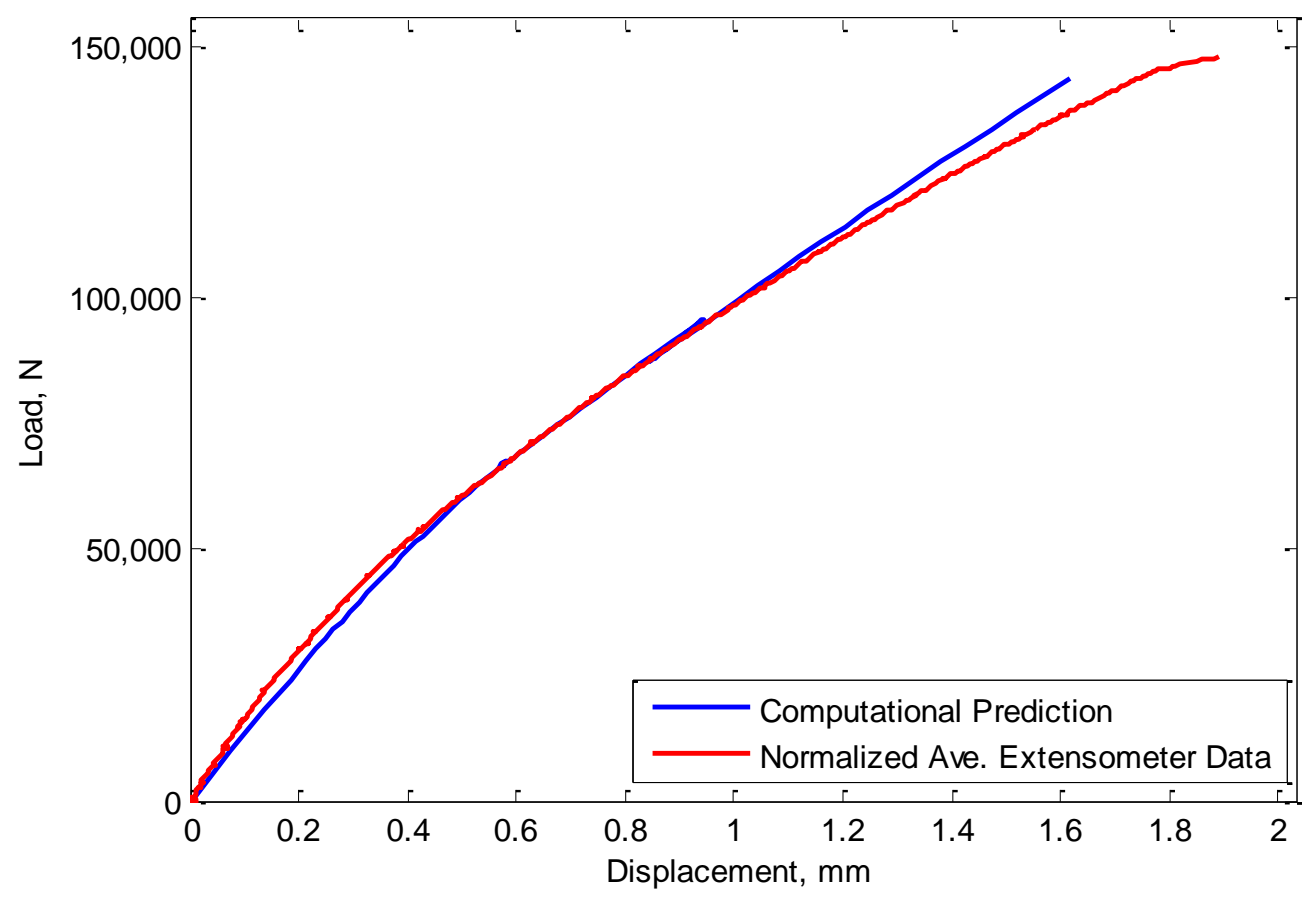

Figure 10. Load-displacement curves for a unitized, stiffened $\pm 60^{\circ} \mathrm{TBC}$ panel without damage or failure modeling. 
As with the TBC 30 case, there is a slight decrease in predicted initial stiffness most likely due to a change in material properties resulting from the VARTM manufacturing process. The experimental buckling load is $21,080 \mathrm{~N}$ compared to the predicted $34,893 \mathrm{~N}$, and the strain-limit load is $148,000 \mathrm{~N}$ compared to the predicted $143,900 \mathrm{~N}$. The imperfection magnitude used to seed the model can influence the buckling load in the Riks step, and a large imperfection may result in buckling loads significantly different than theoretical buckling values. Also, use of a nonlinear material description or damaging material is believed to increase the accuracy of the postbuckling stiffnesses and strain-limit prediction. Corresponding experimental and computational strains are shown in Figure 11.
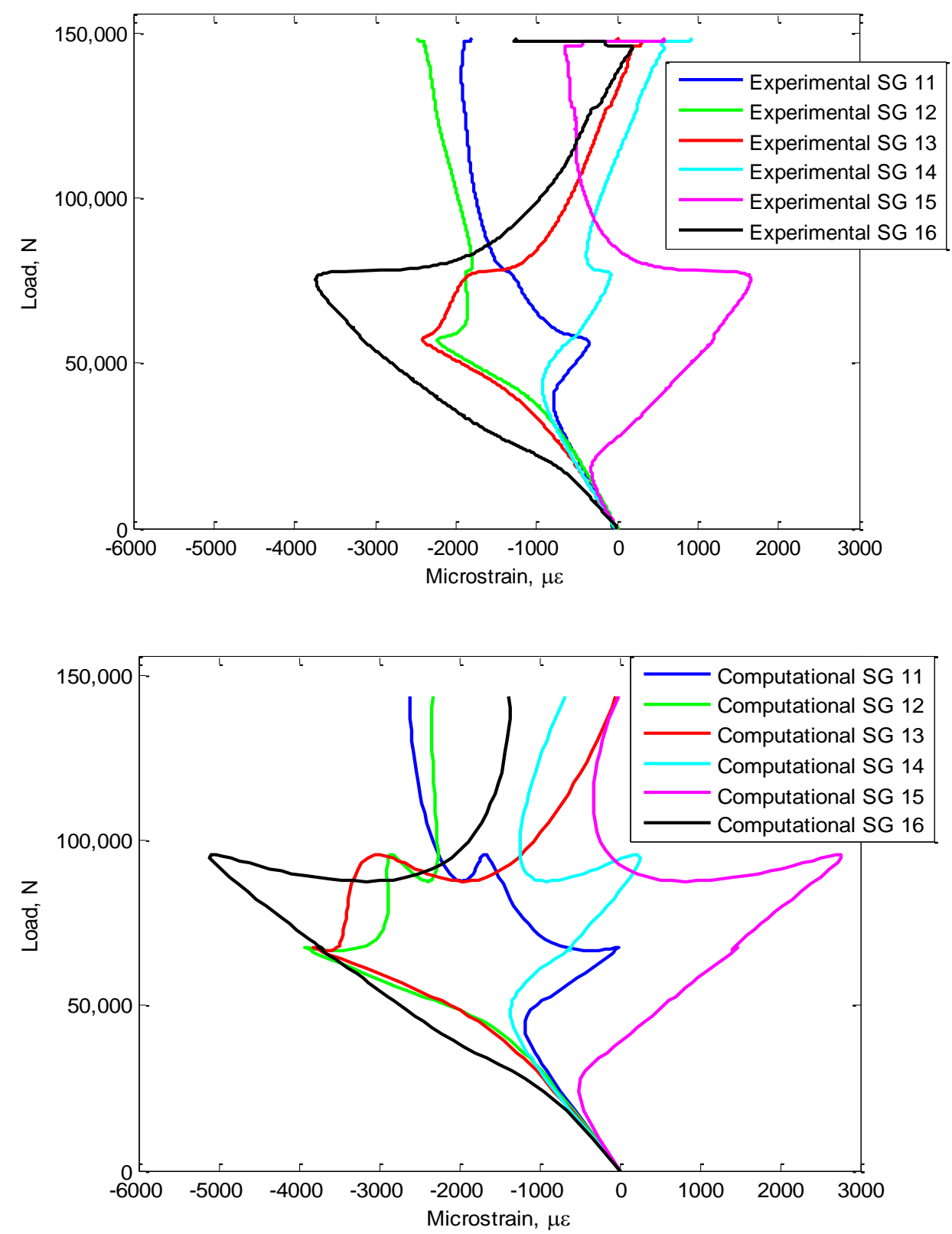

Figure 11. Experimental strains and corresponding computational strains for the TBC 60 specimen demonstrating the mode switch behavior observed experimentally, and the Riks step used to capture such behavior.

The particular strain behavior experimentally observed for the TBC 60 panel is qualitatively captured by the current modeling efforts. However, the loads at which the TBC 60 panel encounters the mode switch is increased in the computational model. Use of a nonlinear material model and measurement of initial geometric imperfections of 
the base panel will both assist in bringing the strain values closer to the observed results, and these aspects are currently being studied.

\section{Conclusion}

This investigation examines the computational buckling and postbuckling response of unitized, stiffened textile fiber reinforced composite plates. Investigations into the global buckling mode shapes, particularly when seeded into a static step, and their affect on postbuckling behavior are made. A two dimensional, tri-axially braided textile is used for the panels, and a new manufacturing approach to creating the stiffener geometry is taken. A study of how the new stiffener formation alters the buckling and postbuckling response away from stiffener debonding is examined, and initial results are provided. A characterization of the VARTM manufacturing process, initial geometrical imperfections, and the resulting material characteristics is significant to capture correct initial behavior, and use of nonlinear material models and/or failure models may increase the agreement between postbuckled stiffnesses and failure loads.

\section{Acknowledgments}

C. J. R. Kosztowny thanks the NASA Office of the Chief Technologist for funding this work through the Space Technology Research Fellowship, as well as the Langley Research Center for providing the means for initial experimental testing. We are also grateful to Dr. Marc Schultz for his interest and involvement in this project.

\section{References}

${ }^{1}$ Jones, R. M. 1998. Mechanics of Composite Materials. CRC Press, pp. 336-344.

${ }^{2}$ Bisagni, C. 2006. "Progressive Delamination Analysis of Stiffened Composite Panels in Post-Buckling," presented at the 47" AIAA/ASME/ASCE/AHS/ASC Structures, Structural Dynamics, and Materials Conference, May 1-4, 2006.

${ }^{3}$ Jegley, D. C. 2009. "Experimental Behavior of Fatigued Single Stiffener PRSEUS Specimens," NASA Langley Research Center, TM - $2009-215955$.

${ }^{4}$ Quek, S. C., A. M. Waas, K. W. Shahwan, and V. Agaram. 2003. "Analysis of 2D triaxial flat braided textile composites," International Journal of Mechanical Sciences, 45: 1077-1096.

${ }^{5}$ Kier, Z. T., A. Salvi, G. Theis, A. M. Waas, and K. Shahwan. 2010. "Predicting Mechanical Properties of 2D Triaxially Braided Textile Composites Based on Microstructure Properties," Department of Aerospace Engineering, University of Michigan, Ann Arbor.

${ }^{6}$ Brush, D. O., and B. O. Almroth. 1975. Buckling of Bars, Plates, and Shells. McGraw-Hill, Inc, pp. 113-116.

${ }^{7}$ Stoll, F. 1994. "Analysis of the Snap Phenomenon in Buckled Plates," International Journal of Non-Linear Mechanics, Vol. 29, No. 2: 123 - 138 . 